\title{
Vibron transport in macromolecular chains with squeezed phonons
}

\author{
D. $\check{C}$ evizović $^{1}$, A. V. Chizhov ${ }^{2,3}$, S. Galović ${ }^{1}$ \\ ${ }^{1}$ Vinča Institute of Nuclear Sciences, 11001 Belgrade, Serbia \\ ${ }^{2}$ Bogoliubov Laboratory of Theoretical Physics, Joint Institute for Nuclear Research, \\ Joliot-Curie, 6, Dubna, 141980, Russia \\ ${ }^{3}$ Dubna State University, Universitetskaya, 19, Dubna, 141980, Russia \\ chizhov@theor.jinr.ru
}

PACS 05.60.Gg, 63.20.-e, 03.65.-w

DOI 10.17586/2220-8054-2018-9-5-597-602

\begin{abstract}
We investigate physical properties of a single vibronic intramolecular excitation propagating through a macromolecule, whose vibrational state can be described as a squeezed vacuum state. For a theoretical description of such a process, the partial dressing method of the vibronic excitation due to its interaction with phonons is used. We study the influence of the model parameters and strength of squeezing on the vibron dressing. It is demonstrated that for certain critical values of the model parameters a polaron crossover can occur, at which there is a sharp change in the migration nature of a vibron from the practically free to the heavy quasiparticle dressed by a phonon cloud. Increasing the strength of phonon squeezing is shown to increase the critical values of the model parameters, so that for high phonon squeezing the polaron crossover takes place in the very strong-coupling and adiabatic regime.
\end{abstract}

Keywords: energy transport, squeezed state, vibron, small polaron.

Received: 12 July 2018

Revised: 21 August 2018

\section{Introduction}

The interest in the use of organic macromolecules (e.g., protein macromolecules, DNA, polymers, and other biological macromolecular structures) in construction of nanostructures, such as nanoparticles, nanocrystals, nanowires, and molecular circuits becomes actual again. Its ability in the field of the miniaturization of microelectronic and optoelectronic devices and, at the same time, its self-assembly capabilities makes them very promising materials in microelectronic and optoelectronic technology [1-3]. The efficient application of such materials requires the knowledge of mechanisms that rule the charge and energy migration along the macromolecule spine at such distances that are comparable to the dimension of the macromolecule. Unfortunately, the formulation of a theoretical model based on the principle of quantum mechanics, which could explain such processes in a satisfactory manner, is not yet complete.

One of the earliest quantum mechanical-based models of the charge and energy transfer in protein molecules was developed by Davydov and his coworkers [4,5]. In these papers Davydov tried to explain the ability of charge and energy transfer at long distances (i.e., along the MC spine) by a soliton model, because the mean life-time of a dressed quasiparticle is much longer than the mean life-time of a bare one. This model was based on the assumption that an external excitation might be captured by a macromolecular chain (MC) due to the interaction with oscillations of the macromolecular chain structure elements. Such a self-trapped (ST) excitation becomes dressed by the phonon cloud and it can move in a soliton form through the macromolecular chain. However, because of the lack of direct experimental evidence for the existence of a soliton in such substances, Davydov's idea has been considered only as an interesting theoretical concept of academic interest. The situation changed when Alexander and Krumhansl [6] suggested that appearance of the so-called unconventional amide-I band observed in crystalline acetanilide (ACN) may be explained in the framework of small-polaron (SP) theories. They supposed that in the process of the vibron-exciton self-trapping a non-adiabatic (small) polaron appears instead of a soliton.

In the polaron theory, the basic energy parameters that determine how the ST particle process will take place and what type it will arise are as follows: the quasiparticle binding energy, the characteristic phonon energy and the energy of quasiparticle-phonon interaction. The standard SP theory works in the case of the strong quasiparticle-phonon interaction [7,8]. But the strength of vibron interaction with the phonon subsystem belongs to an intermediate or even a weak coupling regime [9]. It became clear that the standard SP model cannot be applied to biological macromolecular structures, requiring certain modifications. One way to overcome this problem is to apply the model of the partially dressed SP quasiparticles. Such a method was developed in order to intermediate between the weak and strong coupling limits of exciton-phonon interaction [10-13]. 
When describing the transport mechanism in macromolecular chains, it was usually assumed that the oscillations of their structural elements are due to the thermal fluctuations. However, recent experiments have demonstrated the possibility of transitioning phonon excitations in crystals to a squeezed state [14-16]. Such a phenomenon was achieved due to the action of femtosecond laser pulse radiation in a squeezed state. In particular, this effect was theoretically predicted in Refs. [17-19]. Therefore, it is possible to assume that the state of oscillations of structural elements in the molecular chain can also be brought into a squeezed state under the action of external fields in analogy with crystals.

In this paper we investigate the vibron exciton transport in the framework of partially dressed vibron states as a result of interaction of vibrons with oscillatory excitations of chain structural elements. Here we assume that the quantum state of these excitations are described by the squeezed vacuum state. Special attention is paid to calculation of the dependence of the dressing parameter on the squeezing parameter.

\section{Model}

The model Hamiltonian for describing the single-vibron propagation along a macromolecular chain can be given in a form of the Holstein type [7]:

$$
\begin{aligned}
\hat{H}= & \Delta \sum_{n} \hat{A}_{n}^{\dagger} \hat{A}_{n}-\sum_{n} J_{g} \hat{A}_{n}^{\dagger}\left(\hat{A}_{n+g}+\hat{A}_{n-g}\right)+\sum_{q} \hbar \omega_{q} \hat{B}_{q}^{\dagger} \hat{B}_{q} \\
& +\frac{1}{\sqrt{N}} \sum_{q} \sum_{n} F_{q} \mathrm{e}^{i q n R} \hat{A}_{n}^{\dagger} \hat{A}_{n}\left(\hat{B}_{q}+\hat{B}_{-q}^{\dagger}\right) .
\end{aligned}
$$

Here $\hat{A}_{n}$ is the vibron annihilation operator on the $n$-th lattice site, $\Delta$ is the vibron excitation energy, $J_{g}$ is the hopping constant (i.e. it is the energy of the dipole-dipole interaction of neighboring structure elements on the $\mathrm{MC}), \hat{B}_{q}$ is the phonon annihilation operator with the frequency $\omega_{q}, F_{q}$ is the vibron-phonon coupling parameter, $R$ stands for the distance between two neighboring sites. The transition to the partially dressed polaron picture is achieved by applying the modified Lang-Firsov transformation [10, 12, 13,20]

$$
\hat{U}=\exp \left\{-\frac{1}{\sqrt{N}} \sum_{q} \sum_{n} f_{q} \mathrm{e}^{-i q n R} \hat{A}_{n}^{\dagger} \hat{A}_{n}\left(\hat{B}_{-q}-\hat{B}_{q}^{\dagger}\right)\right\},
$$

where $f_{q}=\delta \cdot F_{q}^{*} /\left(\hbar \omega_{q}\right)$, and $\delta$ is the variational parameter which measures the degree of the vibron dressing $(0 \leq \delta \leq 1)$. By introducing operators of new quasiparticles for dressed vibrons $\hat{a}_{n}=\hat{U} \hat{A}_{n} \hat{U}^{\dagger}\left(\hat{a}_{n}^{\dagger}=\hat{U} \hat{A}_{n}^{\dagger} \hat{U}^{\dagger}\right)$, and new phonons $\hat{b}_{q}=\hat{U} \hat{B}_{q} \hat{U}^{\dagger}\left(\hat{b}_{q}^{\dagger}=\hat{U} \hat{B}_{q}^{\dagger} \hat{U}^{\dagger}\right)$, one gets the transformed Hamiltonian in the form $\hat{\tilde{H}}=\hat{U} \hat{H} \hat{U}^{\dagger}$

$$
\begin{aligned}
\hat{\tilde{H}} & =E \sum_{n} \hat{a}_{n}^{\dagger} \hat{a}_{n}-\sum_{n} J_{g} \hat{a}_{n}^{\dagger}\left(\hat{a}_{n+g} \hat{\Phi}_{n}(g)+\hat{a}_{n-g} \hat{\Phi}_{n}(-g)\right)+\sum_{q} \hbar \omega_{q} \hat{b}_{q}^{\dagger} \hat{b}_{q} \\
& +\frac{1}{\sqrt{N}} \sum_{q} \sum_{n}\left(F_{q}-\hbar \omega_{q} f_{q}^{*}\right) \mathrm{e}^{i q n R} \hat{a}_{n}^{\dagger} \hat{a}_{n}\left(\hat{b}_{q}+\hat{b}_{-q}^{\dagger}\right) \\
& +\frac{1}{N} \sum_{q}\left[\hbar \omega_{q}\left|f_{q}\right|^{2}-F_{q}\left(f_{q}+f_{-q}^{*}\right)\right] \sum_{n \neq n^{\prime}} \mathrm{e}^{i q R\left(n-n^{\prime}\right)} \hat{a}_{n}^{\dagger} \hat{a}_{n} \hat{a}_{n^{\prime}}^{\dagger} \hat{a}_{n^{\prime}},
\end{aligned}
$$

where $E=\Delta-(1 / N) \sum_{q}\left[F_{q}\left(f_{q}+f_{-q}^{*}\right)-\hbar \omega_{q}\left|f_{q}\right|^{2}\right]$ is energy of dressed vibron (which is shifted from the energy of the energy of bare excitation), and

$$
\hat{\Phi}_{n}(g)=\exp \left\{\frac{1}{\sqrt{N}} \sum_{q} f_{q} \mathrm{e}^{-i q n R}\left(\hat{b}_{-q}-\hat{b}_{q}^{\dagger}\right)\left(\mathrm{e}^{-i q R g}-1\right)\right\} .
$$

Defining exciton states in the representation of wave vectors $k$ by $\hat{a}_{k}=(1 / \sqrt{N}) \sum_{n} \mathrm{e}^{i k n R} \hat{a}_{n}$, the Hamiltonian (3) for the single vibron excitation reads:

$$
\begin{aligned}
\hat{\tilde{H}}= & E \sum_{k} \hat{a}_{k}^{\dagger} \hat{a}_{k}+\sum_{q} \hbar \omega_{q} \hat{b}_{q}^{\dagger} \hat{b}_{q}+\frac{1}{\sqrt{N}} \sum_{q} \sum_{k}\left(F_{q}^{*}-\hbar \omega_{q} f_{q}\right) \hat{a}_{k}^{\dagger} \hat{a}_{k+q}\left(\hat{b}_{-q}+\hat{b}_{q}^{\dagger}\right) \\
& -\frac{1}{\sqrt{N}} \sum_{k_{1}} \sum_{k_{2}} J_{g} \hat{a}_{k_{1}}^{\dagger} \hat{a}_{k_{2}}\left\{\mathrm{e}^{-i k_{2} g R} \hat{\Phi}_{k_{1}-k_{2}}(g)+\mathrm{e}^{i k_{2} g R} \hat{\Phi}_{k_{1}-k_{2}}(-g)\right\},
\end{aligned}
$$


where $\hat{\Phi}_{k}(g)=(1 / \sqrt{N}) \sum_{n} \mathrm{e}^{i k n R} \hat{\Phi}_{n}(g)$.

In order to account for the influence of the mechanical oscillation of the chain structure elements on the properties of the ST vibron, we apply the mean-field approach by the averaging of the transformed Hamiltonian over the phonon subsystem. The averaged Hamiltonian obtains the form:

$$
\begin{aligned}
\hat{\tilde{H}}_{M F} & =E \sum_{k} \hat{a}_{k}^{\dagger} \hat{a}_{k}+\frac{1}{\sqrt{N}} \sum_{q} \sum_{k}\left(F_{q}^{*}-\hbar \omega_{q} f_{q}\right) \hat{a}_{k}^{\dagger} \hat{a}_{k+q}\left\langle\hat{b}_{-q}+\hat{b}_{q}^{\dagger}\right\rangle_{p h} \\
& -\frac{1}{\sqrt{N}} \sum_{k_{1}} \sum_{k_{2}} J_{g} \hat{a}_{k_{1}}^{\dagger} \hat{a}_{k_{2}}\left\{\mathrm{e}^{-i k_{2} g R}\left\langle\hat{\Phi}_{k_{1}-k_{2}}(g)\right\rangle_{p h}+\mathrm{e}^{i k_{2} g R}\left\langle\hat{\Phi}_{k_{1}-k_{2}}(-g)\right\rangle_{p h}\right\},
\end{aligned}
$$

in which the symbol $\langle\ldots\rangle_{p h}=\operatorname{Tr}\left\{\hat{\varrho}_{b} \ldots\right\}$ denotes the average over the phonon ensemble.

\section{Squeezed-vacuum phonon state}

The non-classical behavior of phonons upon the action of ultrashort laser pulses on a crystal, as shown in Ref. [21], is due to the generation of biphonon-like states. Such states are characterized by the excitation of phonon modes with equal frequencies and identical in absolute value, but opposite in sign wave vectors, i.e. they are associated with operators $\hat{b}_{q}$ and $\hat{b}_{-q}$. Thus, one can presume that it corresponds to the generation of a multimode squeezed vacuum state of phonons, $|0\rangle_{M S V S}$, which is defined by the unitary operator [22]:

$$
\hat{S}=\exp \left\{\sum_{q}\left[\xi_{q}^{*} \hat{b}_{q} \hat{b}_{-q}-\xi_{q} \hat{b}_{q}^{\dagger} \hat{b}_{-q}^{\dagger}\right]\right\}
$$

with the squeezing parameter $\xi_{q}=r_{q} \mathrm{e}^{i \theta_{q}}$, satisfying the conditions:

so that

$$
\begin{aligned}
& \hat{S}^{\dagger} \hat{b}_{q} \hat{S}=\cosh r_{q} \hat{b}_{q}-\mathrm{e}^{i \theta_{q}} \sinh r_{q} \hat{b}_{-q}^{\dagger}, \\
& \hat{S}^{\dagger} \hat{b}_{q}^{\dagger} \hat{S}=\cosh r_{q} \hat{b}_{q}^{\dagger}-\mathrm{e}^{-i \theta_{q}} \sinh r_{q} \hat{b}_{-q},
\end{aligned}
$$

$$
|0\rangle_{M S V S}=\hat{S}|0\rangle_{b}
$$

where $|0\rangle_{b}$ is the phonon vacuum state, i.e. $\hat{b}_{q}|0\rangle_{b}=0$. Then, for the averages over the phonon ensemble in such a state, we have:

$$
\left\langle\hat{b}_{-q}+\hat{b}_{q}^{\dagger}\right\rangle_{p h} \equiv\left\langle\hat{b}_{-q}+\hat{b}_{q}^{\dagger}\right\rangle_{M S V S}=0,
$$

since $\left\langle\hat{b}_{q}\right\rangle_{M S V S}=\left\langle\hat{b}_{q}^{\dagger}\right\rangle_{M S V S}=0$, and

$$
\left\langle\hat{\Phi}_{k_{1}-k_{2}}(g)\right\rangle_{p h}=\frac{1}{\sqrt{N}} \sum_{n} \mathrm{e}^{i\left(k_{1}-k_{2}\right) n R}\left\langle\hat{\Phi}_{n}(g)\right\rangle_{M S V S},
$$

where the average $\left\langle\hat{\Phi}_{n}(g)\right\rangle_{M S V S}$, as it turns out, does not depend on the site number $n$, i.e.

$$
\left\langle\hat{\Phi}_{n}(g)\right\rangle_{M S V S}=\exp \left\{-\frac{1}{N} \sum_{q}\left|f_{q}\right|^{2}(1-\cos q g R)\left(\cosh 2 r_{q}-\sinh 2 r_{q} \cos \theta_{q}\right)\right\} \equiv \mathrm{e}^{-W_{S V}(g)},
$$

so that

Here the function

$$
\left\langle\hat{\Phi}_{k_{1}-k_{2}}(g)\right\rangle_{p h}=\mathrm{e}^{-W_{S V}(g)} \delta_{k_{1}, k_{2}} .
$$

$$
\begin{aligned}
W_{S V}(g) & =\frac{1}{N} \sum_{q}\left|f_{q}\right|^{2}(1-\cos q g R)\left(\cosh 2 r_{q}-\sinh 2 r_{q} \cos \theta_{q}\right) \\
& =\frac{1}{N} \sum_{q}\left|f_{q}\right|^{2}(1-\cos q g R)\left[\mathrm{e}^{-2 r_{q}} \cos ^{2}\left(\theta_{q} / 2\right)+\mathrm{e}^{2 r_{q}} \sin ^{2}\left(\theta_{q} / 2\right)\right]
\end{aligned}
$$

is positive definite and might play a role of the vibron-band narrowing factor that is caused by the exciton interaction with squeezed phonons.

Thus, the mean-field Hamiltonian (5) takes the form:

$$
\hat{\tilde{H}}_{M F}=\sum_{k} E_{\mathrm{SP}}(k) \hat{a}_{k}^{\dagger} \hat{a}_{k},
$$


with the energy of the small-polaron band state:

$$
E_{\mathrm{SP}}(k)=\Delta-\frac{1}{N} \sum_{q}\left[F_{q}\left(f_{q}+f_{-q}^{*}\right)-\hbar \omega_{q}\left|f_{q}\right|^{2}\right]-2 J_{g} \mathrm{e}^{-W_{S V}(g)} \cos (g k R) .
$$

\section{Degree of small-polaron dressing}

The optimal polaron state should be determined by the minimization of the polaron ground-state energy (15), namely

$$
\mathscr{E}_{G S}=E_{S P}(k=0)=\Delta-\frac{1}{N} \sum_{q}\left[F_{q}\left(f_{q}+f_{-q}^{*}\right)-\hbar \omega_{q}\left|f_{q}\right|^{2}\right]-2 J_{g} \mathrm{e}^{-W_{S V}(g)} .
$$

Consequently, for the fixing of the variational parameter $\delta$ in the unitary transformation (2) the procedure of minimization of the small-polaron ground state energy $\mathscr{E}_{G S}$ is to be used, i.e. $\partial \mathscr{E}_{G S} / \partial \delta=0$ provided $\partial^{2} \mathscr{E}_{G S} / \partial \delta^{2}>0$. Under the assumption that the vibron interacts with optical phonon modes, one can use the dispersionless approximation $\omega_{q}=\omega_{C}$ and $F_{q}=F_{C}$, so that $f_{q}=\delta \cdot F_{C}^{*} /\left(\hbar \omega_{C}\right)$. Then it is convenient to introduce two system dimensionless parameters, namely the adiabatic parameter $B=2\left|J_{g}\right| /\left(\hbar \omega_{C}\right)$ and the coupling constant $S=E_{\mathrm{B}} /\left(\hbar \omega_{C}\right)$ (where $E_{\mathrm{B}}=(1 / N) \sum_{q}\left\{\left|F_{q}\right|^{2} /\left(\hbar \omega_{q}\right)\right\}=\left|F_{C}\right|^{2} /\left(\hbar \omega_{C}\right)$ is the lattice deformation energy). These parameters are very convenient for describing the polaron properties. The adiabatic parameter $B$ determines the character of the lattice deformation engaged in the polaron formation, while the coupling constant $S$ (which was originally introduced by Holstein) determines the polaron spatial size [7,8] Thus the problem of minimization of $\mathscr{E}_{G S}$ reduces effectively to minimization of the function

$$
\mathscr{E}=-S(2-\delta) \delta-B \mathrm{e}^{-\delta^{2} W_{S V}(S)},
$$

where $\mathscr{E}$ is the polaron ground state energy normalized to the characteristic phonon energy $\hbar \omega_{C}$. The first therm in Eq. (17) corresponds to the polaron binding energy, while the second one corresponds to the width of the quasiparticle energy band. The vibron-band narrowing factor (13) in the case when $r_{q}=r$ and $\theta_{q}=\theta$ takes the form

$$
W_{S V}(S)=S(\cosh 2 r-\sinh 2 r \cos \theta)=S\left[\mathrm{e}^{-2 r} \cos ^{2}(\theta / 2)+\mathrm{e}^{2 r} \sin ^{2}(\theta / 2)\right] .
$$

It is worth noting that the vibron-band factor (18) for squeezed phonons is principally different from the analogous factor for thermal phonons [12]. This is due to the fact that with an appropriate choice of squeezing phase, e.g., when $\theta=0$ and Eq. (18) has the form $W_{S V}(S)=S \exp (-2 r)$, for a fixed parameter $S$ the narrowing factor can be made arbitrarily small for large values of the strength of squeezing $r$, while the narrowing factor for thermal phonons is limited by the value $W_{T}(S)=S$ at zero temperature. Thus, by choosing the phase and the strength of squeezing of phonons in a proper way, it opens up new opportunities for control of the character of energy transport through macromolecular chains.

\section{Results}

Here we consider the case of a quasi 1D macromolecular chain, so we take the parameter $g$ equal to 1 that corresponds to the interaction of the nearest neighboring structural elements of the chain. In our theoretical analysis we considered the case when $\theta=0$ in order to explore the parameter region inaccessible to the case of thermal phonons. For the strength of phonon squeezing we have chosen the following three values, namely $r=0.1,0.2$, and 0.4, although recent experiments with crystals generate rather moderate degrees of squeezing of phonon states. The results of minimization of Eq. (17) for various model parameters in terms of $B, S$, and the strength of phonon squeezing $r$ are plotted in Fig. 1 and Fig. 2.

In these figures, one can see that for various values of the model parameters there are two different regimes of the dressed vibron nature. In the first regime, vibron dressing changes continuously with changing the model parameters, while in the second regime it undergoes an interrupted transition for certain values of model parameters. Additionally, we can see that the strength of phonon squeezing significantly influences these processes.

More precise details of vibron properties depending on the model parameters are given in Fig 2. Here one can remark that in the non-adiabatic regime (i.e., for small values of $B$ ) vibron dressing increases continuously with increasing strength of the exciton-phonon coupling $S$. In this regime an exciton is a strongly dressed quasiparticle, even in the case of a weak exciton-phonon coupling regime. At the same time, the ground state energy of the vibron decreases continuously with increasing $S$. This dependence is typical for standard non-adiabatic polarons (where the process of the exciton dressing is quite adequately described by the standard Lang-Firsov approach [20]). With an increase in $B$, the initial value of the dressing parameter decreases, but with increasing $S$ it smoothly increases and approaches its maximum value equal to unity (i.e., exciton becomes fully dressed by a phonon cloud). With 


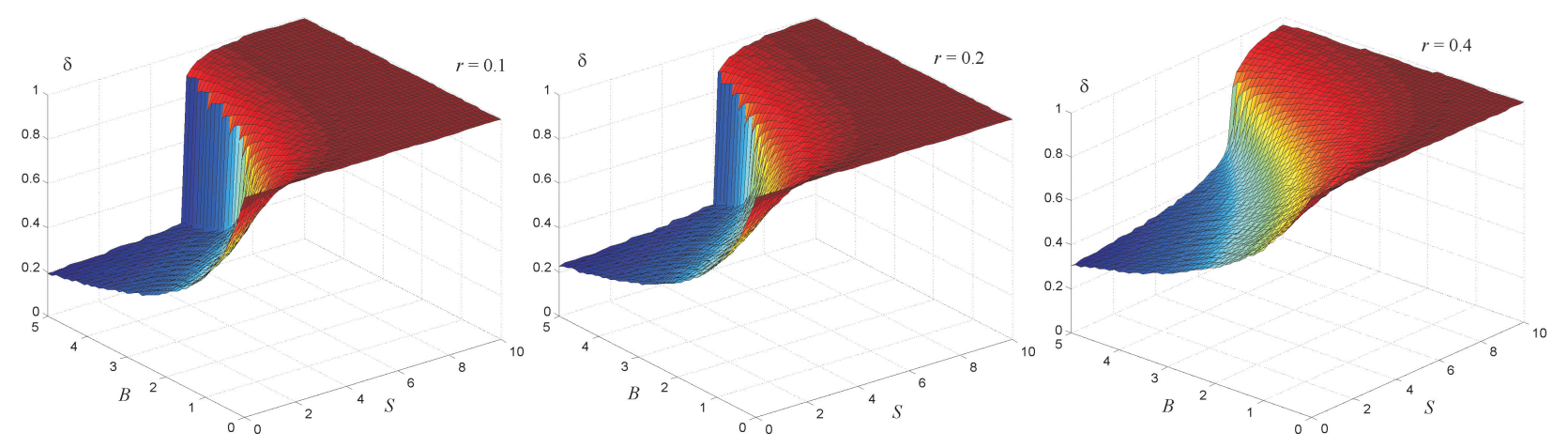

FIG. 1. Dressing fraction $\delta$ as a function of the adiabatic parameter $B$ and the coupling constant $S$ for various strengths of squeezing $r(\theta=0)$
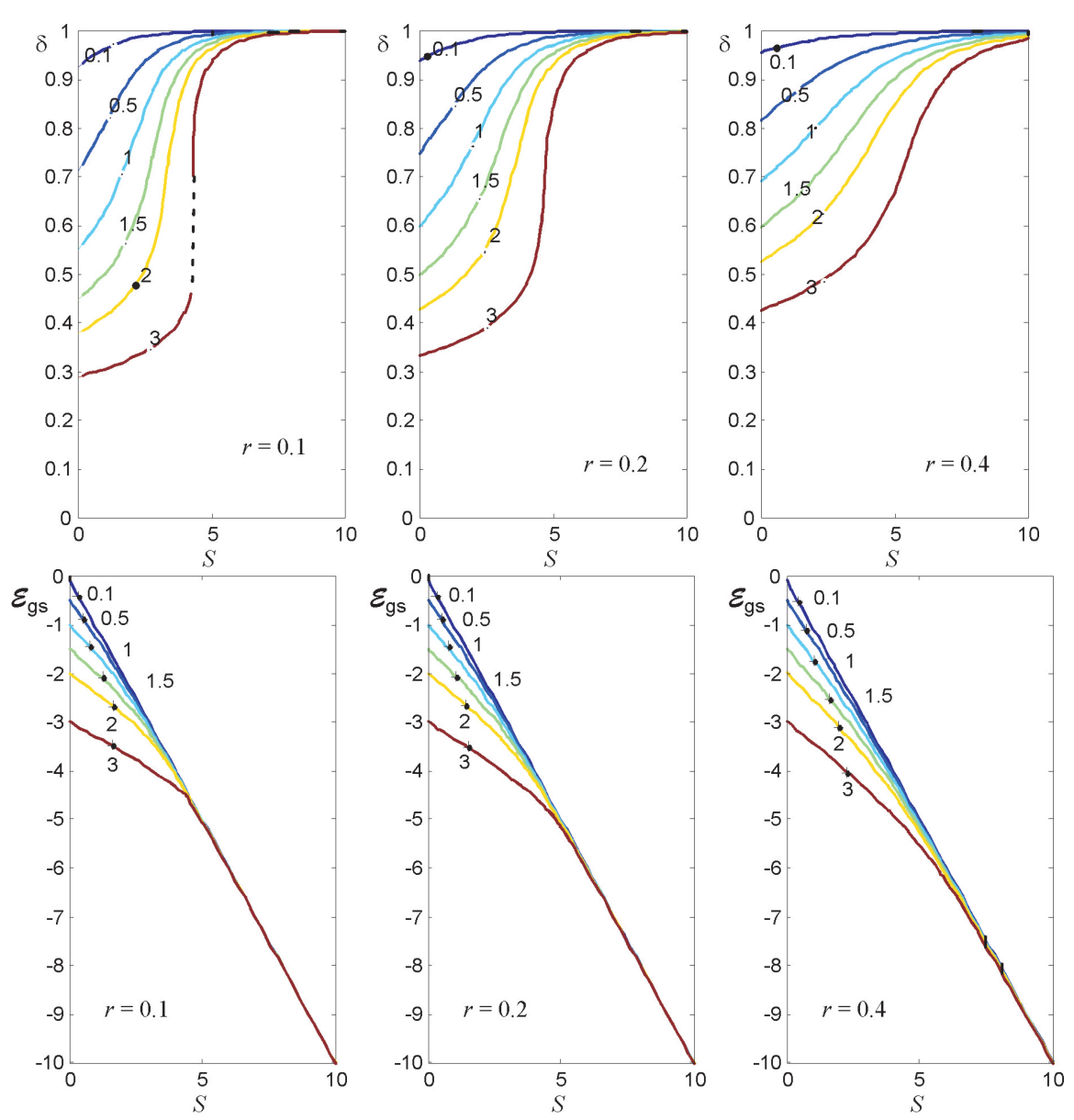

FIG. 2. Dressing fraction $\delta$ (first row) and polaron ground-state energy $\mathscr{E}_{G S}$ (second row) as functions of the coupling constant $S$ for various values of the adiabatic parameter $B$ (indicated on the curves) and the strengths of squeezing $r(\theta=0)$

a further increase in $B$, it reaches such a critical value $B_{C}$ for which $\delta$ becomes a discontinuous function of $S$ for the critical value $S_{C}$ and suffers interruption (such a behavior of the dressing fraction $\delta$ at the critical point is represented by a dashed curve in the left plot of the first row of Fig. 2). At the same time, at the critical point $\mathscr{E}_{G S}$ ceases to be a smooth function of $S$ and has a break (lower curve in the left plot of the second row of Fig. 2). With a further increase in $B$, the excitation becomes slightly dressed and enters the adiabatic regime. 
On the other hand, the effect of increasing $B$ can be moderated by an increase in the value of the parameter $r$. As a consequence, with the help of increasing the strength of squeezing it is possible to influence the increase in the system adiabaticity and to keep it in the model-parameter area, that is characteristic for non-adiabatic systems.

Figure 1 shows that there is a whole set of critical values of the model parameters $\left(S_{C}, B_{C}\right)$ under which the polaron crossover occurs. With such a crossover there appears a sharp change in the migration nature of a vibron from the practically free to the heavy quasiparticle "dressed" by a phonon cloud. These critical values of the adiabatic parameter $B_{C}$ and the coupling constant $S_{C}$ increase with increasing the strength of phonon squeezing. It means that for high phonon squeezing the polaron crossover takes place in a very strong-coupling and adiabatic regime.

\section{Conclusion}

We have studied propagation of a single vibronic intramolecular excitation along a 1D macromolecule under the assumption that its vibrational state is described by a squeezed vacuum state. Using the partial dressing method of the vibronic excitation due to its interaction with phonons, for various model parameters and the degree of phonon squeezing we have demonstrated the existence of a polaron crossover, at which a sudden change in the migration nature of a vibron from the practically free to the heavy quasiparticle dressed by a phonon cloud occurs. Increasing the strength of phonon squeezing is shown to increase the crossover critical values of the model parameters, so that for high phonon squeezing the polaron crossover takes place in the very strong-coupling and adiabatic regime. With increasing the strength of squeezing, the crossover critical values of the adiabatic parameter and the coupling constant were shown to increase, so that for high phonon squeezing the polaron crossover takes place in the very strong-coupling and adiabatic regime.

\section{Acknowledgements}

This work is partly supported by the bilateral project between the Serbian Ministry of Education and Science and "Theory of Condensed Matter" at JINR, Dubna, and by Serbian Ministry of Education and Science, under Contract Nos. III-45010 and III-45005.

\section{References}

[1] Dekker C., Ratner M. A. Electronic properties of DNA. Phys. World, 2001, 14 (8), P. 29-34.

[2] Convell E. Polarons and transport in DNA. Top. Curr. Chem., 2004, 237, P. 73-102.

[3] Mirkin C. A., Letsinger R. L., Mucic R. C., Storhoff J. J. A DNA-based method for rationally assembling nanoparticles into macroscopic materials. Nature, 1996, 382 (6592), P. 607-609.

[4] Davydov A. S. The theory of contraction of proteins under their excitation. J. Theor. Biol., 1973, 38 (3), P. $559-569$

[5] Davydov A. S. Solitons in molecular systems. Phys. Scr., 1979, 20 (2), P. 387-394.

[6] Alexander D. M., Krumhansl J. A. Localized excitations in hydrogen-bonded molecular crystals. Phys. Rev. B, 1986, 33 (10), P. $7172-7185$.

[7] Holstein T. Studies of polaron motion: Part I. The molecular-crystal model. Annals of Physics, 1959, 8 (3), P. $325-342$.

[8] Rashba E. I. Self-trapping of excitons. Excitons, North-Holland, Amsterdam, 1982, P. 543-602.

[9] Pouthier V. Vibron phonon in a lattice of H-bonded peptide units: A criterion to discriminate between the weak and the strong coupling limit. J. Chem. Phys., 2010, 132 (3), 035106.

[10] Brown D.W., Ivić Z. Unification of polaron and soliton theories of exciton transport. Phys. Rev. B, 1989, 40 (14), P. 9876-9887.

[11] Yarkony D., Silbey R. Comments on exciton phonon coupling: Temperature dependence. J. Chem. Phys., 1976,65 (3), P. $1042-1052$.

[12] Čevizović D., Galović S., Ivić Z. Nature of the vibron self-trapped states in hydrogen-bonded macromolecular chains. Phys. Rev. E, 2011, 84 (1), 011920.

[13] Čevizović D., Galović S., Reshetnyak A., Ivić Z. Vibron self-trapped states in biological macromolecules: Comparison of different theoretical approaches. J. Phys.: Conf. Ser., 2012, 393 (1), 012033.

[14] Garret G. A., Rojo A. G., Sood A. K., Whitaker J. F., Merlin R. Vacuum squeezing of solids: Macroscopic quantum states driven by light pulses. Science, 1997, 275 (5306), P. 1638-1640.

[15] Beaud P. et al. Spatiotemporal stability of a femtosecond hard-X-ray undulator source studied by control of coherent optical phonons. Phys. Rev. Lett., 2007, 99 (17), 174801.

[16] Johnson S.L. et al. Directly observing squeezed phonon states with femtosecond X-ray diffraction. Phys. Rev. Lett., 2009, 102 (17), 175503 .

[17] Nazmitdinov R. G., Chizhov A. V. Effect of compressed light on pumping of a crystal. Pis'ma v ZhETF, 1990,52 (7), P. $993-996$.

[18] Artoni M., Birman J. L. Non-classical states in solids and detection. Opt. Commun., 1994, 104 (4-6), P. 319-324.

[19] Hu X., Nori F. Quantum phonon optics: Coherent and squeezed atomic displacements. Phys. Rev. B, 1996, 53 (5), P. $2419-2424$.

[20] Lang I. G., Firsov Yu. A. Kinetic theory of semiconductors with low mobility. ZhETF, 1962, 43 (5/11), P. 1843-1860.

[21] Misochko O. V., Hu J., Nakamura K. G. Controlling phonon squeezing and correlation via one- and two-phonon interference. Phys. Lett. A, 2011, 375 (46), P. 4141-4146.

[22] Loudon R., Knight P. L. Squeezed light. J. Mod. Opt., 1987, 34 (6-7), P. 709-759. 